\title{
A method for atomic force microscopy cantilever stiffness calibration under heavy fluid loading
}

\author{
Scott J. Kennedy, ${ }^{1, a)}$ Daniel G. Cole, ${ }^{2}$ and Robert L. Clark ${ }^{3}$ \\ ${ }^{1}$ Department of Mechanical Engineering and Materials Science, Center for Biologically Inspired Materials \\ and Material Systems, Duke University, Durham, North Carolina 27708, USA \\ ${ }^{2}$ Department of Mechanical Engineering and Materials Science, Swanson School of Engineering, \\ University of Pittsburgh, Pittsburgh, Pennsylvania 15260, USA \\ ${ }^{3}$ Hajim School of Engineering and Applied Sciences, University of Rochester, Rochester, \\ New York 14627, USA
}

(Received 21 January 2009; accepted 24 October 2009; published online 7 December 2009)

\begin{abstract}
This work presents a method for force calibration of rectangular atomic force microscopy (AFM) microcantilevers under heavy fluid loading. Theoretical modeling of the thermal response of microcantilevers is discussed including a fluid-structure interaction model of the cantilever-fluid system that incorporates the results of the fluctuation-dissipation theorem. This model is curve fit to the measured thermal response of a cantilever in de-ionized water and a cost function is used to quantify the difference between the theoretical model and measured data. The curve fit is performed in a way that restricts the search space to parameters that reflect heavy fluid loading conditions. The resulting fitting parameters are used to calibrate the cantilever. For comparison, cantilevers are calibrated using Sader's method in air and the thermal noise method in both air and water. For a set of eight cantilevers ranging in stiffness from 0.050 to $5.8 \mathrm{~N} / \mathrm{m}$, the maximum difference between Sader's calibration performed in air and the new method performed in water was $9.4 \%$. A set of three cantilevers that violate the aspect ratio assumption associated with the fluid loading model (length-to-width ratios less than 3.5) ranged in stiffness from 0.85 to $4.7 \mathrm{~N} / \mathrm{m}$ and yielded differences as high as 17.8\%. (C) 2009 American Institute of Physics. [doi:10.1063/1.3263907]
\end{abstract}

\section{INTRODUCTION}

The ratio between force and displacement at the tip of a rectangular cantilever beam is a simple function of the Young's modulus and geometry of the beam. However, in the case of a microscopic cantilever this relationship is obscured by uncertainty in the beam material and geometric properties. These uncertainties stem from limitations in the photolithographic processes used to manufacture microcantilevers. While these techniques enable the manufacturing of cantilever beams less than $1 \mu \mathrm{m}$ in thickness, the thickness tolerance resulting from these methods is such that manufactures publish stiffness specification ranges that can vary by as much as a factor of 5 from the nominal value.

Since atomic force microscopy (AFM) cantilevers cannot be manufactured with precisely known stiffnesses, they must be calibrated before they can be used to make quantitative force measurements. A great deal of effort has been spent pursuing calibration techniques. ${ }^{1-10}$ Some calibration techniques are cumbersome, involving procedures that are comparable in complexity to the experiments that require the calibrated cantilevers. ${ }^{1,5,11,12}$ Other techniques require only the measurement of the thermally driven, random vibration of a cantilever and perhaps knowledge of a few of the parameters characterizing the cantilever and its fluid environment. ${ }^{2,13,14}$ The majority of the exploration into cantilever calibration techniques has been validated through the

\footnotetext{
${ }^{a)}$ Electronic mail: scott.kennedy@duke.edu.
}

comparison of one technique to another. While there have been some comparisons between existing calibration techniques and calibrations traceable to international standards, ${ }^{7}$ more work is needed to determine the accuracy of various techniques in various situations.

Currently, two of the most popular cantilever calibration methods are the thermal noise method ${ }^{13}$ and Sader's method. ${ }^{14}$ The first method is based on equipartition theory, which relates the stiffness to the magnitude of the free vibration of a cantilever, while the second incorporates a model of the fluid dynamics and is based on identifying features of the free vibration dynamics. Calibrations performed in air with these techniques vary in their agreement from case to case, with some researchers finding excellent agreement. ${ }^{9}$ Typically, researchers find that these method agree to within $20 \%$. ${ }^{7,15}$ Additionally, there can be variation between calibrations performed on the same cantilever based on data collected in both air and water. ${ }^{2,16}$ There should be no dramatic change in cantilever stiffness caused by changing the fluid environment from air to water. Changes in the resonant frequency correlating to a change in the surface stress on a cantilever have been observed; ${ }^{17}$ however, the changes are small compared with the frequency shift caused by the mass loading effect that a liquid such as water has on a beam and the mechanism driving these changes is still debated. ${ }^{18}$

As will be discussed in Sec. II, the thermal noise method and Sader's method are fundamentally different in ways that yield different strengths and weaknesses. For this reason, it can be beneficial to use both techniques as a way to detect 
improper calibration conditions that impact the two methods differently. For example, an error impacting the scale of an AFM deflection measurement will affect the thermal noise method, but not the Sader method. Like the Sader method, the liquid environment calibration method presented here is based on the cantilever-fluid dynamics allowing a check against the thermal noise method. The Sader method is specifically designed for light fluid loading situations only, ${ }^{14}$ while the thermal noise method presented by Hutter and Bechhoeffer ${ }^{13}$ is equally valid under any amount of fluid loading. The fluid-loading-based calibration technique for liquid environments presented here creates an additional comparison point. With this additional technique it is possible to compare calibration techniques based on both the physics of the equipartition theorem and the fluid loading model in gas or in liquid environments. While this comparison alone cannot establish accuracy, it can serve as a useful tool to researchers that do not have access to artifacts traceable to international standards.

Enabling a comparison of calibration techniques without the need for data collected in air would be beneficial to biomolecular researchers. One of the exciting fields of AFM usage is the application of AFM to biological molecules. ${ }^{19} \mathrm{In}$ most cases, this requires AFM operation in liquid environments. While it is acceptable to calibrate a cantilever in air before using it in liquid, it is more desirable to calibrate an AFM cantilever in situ. Air and liquid test setups generally require different hardware and it is best to handle the fragile cantilevers as little as possible.

Advances in liquid environment calibration are also critical if microcantilevers are to be incorporated as chemical or biological sensors in a "laboratory on a chip" device. Proper characterization of the thermal vibrations of a cantilever in air and in water requires different measurement parameters, specifically, different sample rates and magnitude ranges to accommodate the different system dynamics. Requiring a laboratory on a chip to acquire data over multiple frequency and magnitude ranges adds to the complexity and cost of the design. Additionally, heavy fluid loading calibration would allow periodic recalibration in situ to check the status of cantilevers that could be deployed as monitors of liquid environments.

Another enabling aspect of a new heavy fluid loading technique has already been demonstrated in air. A common way to calibrate the cantilever deflection measurement sensor [referred to as the inverse optical lever sensitivity (InvOLS) (Ref. 20)] is to apply a static displacement to the cantilever with a calibrated actuator. Alternately, Sader's method can be used to determine the stiffness of a cantilever, and equipartition theorem can then be used to determine the InvOLS using only noncontact data. ${ }^{21} \mathrm{~A}$ new heavy fluid loading technique could be used in a similar fashion to extend both noncontact force and displacement calibration to liquid environments. This would be advantageous in research situations where soft cantilever or surface coatings inhibit the ability to perform static force InvOLS calibrations or where there is concern that the static deflection process might damage the cantilever tip. ${ }^{21}$ It would also enable calibration in laboratory on a chip designs where a calibrated actuator may not be available.

\section{BACKGROUND}

Some calibration techniques involve experiments specifically designed for the sole purpose of measuring the stiffness (e.g., pushing against a structure of known stiffness or adding a known mass to the tip of the cantilever and observing the change in resonant frequency. ${ }^{11,12}$ ) In 1993, Hutter and Bechhoefer ${ }^{13}$ equated the available thermal energy to the observed random vibration of a cantilever as defined by the equipartition theorem. The method has been widely adopted by the scanning probe microscopy community as the theory applies broadly to microstructures including both rectangular and V-shaped cantilevers. However, variations between calibrations performed in air and in water or between the thermal noise method and calibrations traceable to international standards on the order of $10 \%-20 \%$ have been reported. ${ }^{2,7}$ Some of the discrepancies may stem from complications associated with the process of determining the InvOLS of an AFM. ${ }^{21}$ Other issues have been characterized and can be accounted for such as the difference in beam shape between a dynamically oscillating beam and a statically deflected beam, the division of energy between cantilever bending modes, and the laser spot size and position along the length of a cantilever in the case of an optical lever AFM. ${ }^{20,22}$ Another source of error that can impact this method stems from noise that can be misinterpreted to be thermally driven cantilever motion. Noise can arise from acoustic, electrical, or mechanical vibrations in the local environment that can impact the AFM system. To reduce the impact of system noise on a stiffness calculation, a simple harmonic oscillator ( $\mathrm{SHO}$ ) model can be fit to the frequency response of the beam around the resonant peak where noise typically has the least impact as an approximation for the thermal response of the cantilever in the absence of system noise.

In 1998, Sader $^{23}$ improved upon the existing models of microcantilever dynamics by adding the mass loading and damping induced by the fluid enveloping the cantilever to the in vacuo cantilever equations of motion. Sader also introduced an expression for the stiffness of rectangular cantilevers based on first resonance parameters that took this fluid loading model into account. ${ }^{14}$ Proper application of this technique requires that the quality factor of the cantilever-fluid system is high $(Q \gg 1)$. As a result, this technique is generally limited to calibrating microcantilevers in gas environments. Additionally, the fluid loading model assumes that the length of the beam greatly exceeds the width of the beam. ${ }^{23}$ This assumption places restrictions on the cantilevers that can be calibrated with techniques based on this loading including both Sader's calibration technique and the new technique presented here. Sader's technique possesses two drawbacks in that it cannot be used with V-shaped cantilevers or in liquid environments; however, it has the significant advantage of not requiring that data be accurately scaled. Calibration is based on the shape of the power spectral density (PSD), not the magnitude. 
The modeled thermal response of the cantilever has been further refined by applying the fluctuation-dissipation theorem to the fluid loaded cantilever model. ${ }^{24,25}$ More recently, the fluid-cantilever interaction and thermal response have been formulated in terms analogous to a feedback system where the fluid loading acts like a feedback mechanism to the cantilever motion. ${ }^{25}$ This approach allows for the cantilever response to be modeled based on the cantilever length and width, the properties of the fluid environment, the natural frequency of the cantilever, and a fluid-to-cantilever mass ratio. While the scaling of this model satisfies equipartition theorem, the heavy fluid loading technique presented here is similar to Sader's calibration method in that it does not require the PSD to be accurately scaled. For this reason, it is also a noncontact calibration method. Like Sader's model, it cannot be used to describe V-shaped cantilever dynamics.

\section{THEORY}

Cantilever stiffness calibration techniques based on the analysis of the random thermally driven vibrations of a free cantilever are nondestructive and do not require any equipment in addition to what is normally required to make AFM measurements. This makes calibration techniques based on the thermal response highly desirable and a great deal of work has been aimed at improving such methods. This research effort has focused on such techniques.

\section{A. Parameter based stiffness estimation}

There have been multiple efforts to estimate cantilever stiffness using various parameters that characterize the thermal vibrations of a cantilever. These parameters can be obtained through curve fitting models such as a SHO model or by identifying the appropriate points directly from frequency response plots (e.g., frequency response plots can be used to identify the resonant frequency $\omega_{r}$ and the quality factor $Q$ through the half-power bandwidth method ${ }^{26}$ ).

In 1999, Sader published a parameter based calibration that takes into account the fluid loading of the surrounding environment on the cantilever. ${ }^{14}$ In this technique the stiffness is estimated as

$$
k=0.1906 \rho b^{2} L Q \Gamma_{i}\left(\omega_{r}\right) \omega_{r}^{2},
$$

where $L$ and $b$ are the cantilever length and width, $\rho$ is the density of the surrounding fluid, and $\Gamma_{i}$ is the imaginary part of the function describing the hydrodynamic loading on the beam evaluated at the resonance frequency $\omega_{r}$. This hydrodynamic function was presented in an earlier paper by Sader $^{23}$ where he used the Euler-Bernoulli beam equation to determine cantilever dynamics and then added the fluid loading to the beam model. Sader's calibration method is used for comparison to the new method presented in this paper.

\section{B. Fluctuation-dissipation theorem and fluid-structure interaction}

Another refinement to microcantilever thermal response modeling was the application of the fluctuation-dissipation theorem. Previous models treated thermal excitation as white noise. In 2004, Paul $^{24}$ modeled the thermal excitation of a microcantilever applying the results of the fluctuationdissipation theorem to a fluid-loaded cantilever model. The fluctuation-dissipation theorem dates back to 1905 when Einstein $^{27}$ theorized that Brownian motion and the drag on a particle forced to move through a fluid arose from the same mechanism. This implies that thermal noise of a particle or structure is not white, rather it is a frequency dependant function of the motility of that particle or structure. This adjustment to the thermal excitation has been used in a few studies. ${ }^{24,25,28}$ One interesting result of the application of the fluctuation-dissipation theorem to a microcantilever is that the thermal response has zero magnitude at dc. This is in agreement with the expected behavior of a microcantilever. The SHO model assuming white noise discussed previously contains a nonzero value at $\mathrm{dc}$. This term is important in the formulation of the SHO model and it is even used as a parameter in a calibration estimation technique. ${ }^{29}$ However, a nonzero thermal response at dc indicates a constant deflection. It would imply that the thermal excitation results in cantilever drift that is not restored by the cantilever stiffness. While there are other elements in an AFM system that can lead to cantilever position measurement drift and the bimetallic effect can induce temperature dependant static deflections of cantilevers, the thermal response of the cantilever deflection at dc should be zero as the fluctuation-dissipation theorem predicts. Cole ${ }^{25}$ incorporates the fluctuationdissipation theorem in a fluid-structure interaction (FSI) formulation that treats the hydrodynamic forces as a feedback mechanism. The equation of motion for the in vacuo cantilever relating its mass $m$, modal stiffness $k_{n}$, and model deflection $q_{n}$ is

$$
m \ddot{q}_{n}+k_{n} q_{n}=k_{n} \tilde{q}_{n} .
$$

The system input is characterized in terms of a modal fluctuating displacement $\widetilde{q}_{n}$, which is the ratio of the thermally driven random modal force $\tilde{f}_{n}$ to the modal stiffness of the cantilever $k_{n}$, such that $\tilde{f}_{n}=k_{n} \widetilde{q}_{n}$.

The damping intrinsic to the beam is assumed to be trivial relative to the damping induced by the surrounding fluid. The resulting transfer function of the in vacuo cantilever is

$$
G(s)=\frac{q_{n}(s)}{\widetilde{q}_{n}(s)}=\frac{\omega_{n}^{2}}{s^{2}+\omega_{n}^{2}},
$$

where $\omega_{n}$ is the cantilever natural frequency. The hydrodynamic loading on the cantilever is a response to cantilever motion; it is therefore feedback. The hydrodynamic function used by Sader describes this feedback in the following controller $K$ :

$$
K(s)=\gamma \frac{s^{2}}{\omega_{n}^{2}} \Gamma(s),
$$

where $\gamma$ is a mass ratio between the cantilever and the surrounding fluid,

$$
\gamma=\frac{m^{\prime}}{m},
$$

where $m$ is the cantilever mass, and 


$$
m^{\prime}=\frac{\pi b^{2} L \rho}{4} .
$$

It is important to note that due to a difference in convention, the hydrodynamic functions as presented by Sader and by Cole are complex conjugates of each other, and care must be taken to ensure consistent convention in the combination of Sader's hydrodynamic correction function and Cole's formulation. The closed-loop system $P$, which includes both the in vacuo dynamics and the hydrodynamic feedback, is

$$
P(s)=\frac{q_{n}(s)}{\widetilde{q}_{n}(s)}=\frac{\omega_{n}^{2}}{s^{2}+\omega_{n}^{2}+\gamma s^{2} \Gamma(s)} .
$$

Finally, applying the fluctuation-dissipation theorem yields the modal PSD of the thermal response

$$
S_{q q, n}(\omega)=-2 k_{B} T \frac{\operatorname{Im}[P(j \omega)]}{m \omega_{n}^{2} \omega} .
$$

This result is scaled by the temperature $T$ and the Boltzmann constant $k_{B}$ as required by the equipartition theorem. The sum of the modal PSDs scaled by the modal displacements yields the total dynamic response at the tip of the beam,

$$
S_{w w}(\omega)=\sum \phi_{n}^{2} S_{q q, n}(\omega),
$$

where $\phi_{n}$ is the mode shape $\phi_{n}(x)$ evaluated at the tip of the beam. The mode shapes are the solutions for transverse waves within a Euler-Bernoulli beam and are described as follows:

$$
\begin{aligned}
\phi_{n}(x)= & \cos \left(\alpha_{n} x\right)-\cosh \left(\alpha_{n} x\right)+\left[\sinh \left(\alpha_{n} x\right)\right. \\
& \left.-\sin \left(\alpha_{n} x\right)\right] \frac{\cos \left(\alpha_{n} L\right)+\cosh \left(\alpha_{n} L\right)}{\sin \left(\alpha_{n} L\right)+\sinh \left(\alpha_{n} L\right)} .
\end{aligned}
$$

To satisfy the boundary conditions for a cantilever beam, the wave number $\alpha_{n}$ must solve the equation

$$
-1=\cos \left(\alpha_{n} L\right) \cosh \left(\alpha_{n} L\right) \text {. }
$$

Therefore, $\alpha_{1} L \approx 1.875$ and $\alpha_{2} L \approx 4.694$. The wave number relates the cantilever length to its modal frequencies, mass, and modal stiffnesses; it appears both in the fluid-structure interaction model and in the calibration technique presented here.

\section{METHODS}

\section{A. Data acquisition and processing}

The cantilever response data were collected using an Asylum Research MFP-3D AFM system. The data were acquired according to the in-program cantilever stiffness calibration guidelines. Photodiode calibrations were determined by averaging the slopes of the voltage-position curves for both the approaches and retractions for five contact cycles between the cantilevers and a silicon substrate. Each calibration was performed five times and averaged. The plan view dimensions, tip height, and the distance from the tip to the cantilever end were measured with an optical microscope.

The frequency response data were processed in MATLAB. To account for the difference between the slope-to-position ratios of the cantilever end under static loading (during calibration) and freely vibrating (during thermal data collection), the appropriate correction factor $\chi$ was found according to Ref. 20. In this study, $\chi$ values ranged from 1.027 to 1.082 . (In the limit of an infinitely small laser spot, positioned exactly at the end of a rectangular cantilever, $\chi=1.09$.) To compare the intrinsic cantilever stiffness identified by Sader's technique and the new technique presented here with the effective cantilever stiffness identified by the thermal noise method, corrections were made to account for the cantilever angle, the tip height, and the distance from the tip of the probe to the end of the cantilever. ${ }^{30,31}$

\section{B. Data fitting techniques}

The fluid-structure interaction model of cantilever thermal response was fit to the measured data by minimizing a cost function that compared measured data with the theoretical response corresponding to a given set of input parameters. The hydrodynamic loading used within the model, $\Gamma(i \omega)$, was determined using the measured specifications for the cantilever lengths and widths, and the room temperature properties of the fluids enveloping and exciting the cantilevers; it was assumed that these parameters are known with sufficient accuracy. The model fitting process did not assume knowledge of parameters such as the thickness and the Young's modulus, which cannot be known with sufficient accuracy due to the manufacturing methods. The remaining parameters needed to model the thermal response were the natural frequency $\omega_{n}$ and the fluid-to-cantilever mass ratio $\gamma$. Additionally, parameters were fit to account for measurement noise and a fitting parameter was added to allow the magnitude of the model to be rescaled. This "scaleless" fitting was performed in response to the observation that the fitting process was extremely sensitive to the magnitude of the data, which is subject to the inaccuracy of the photodiode calibration process. For a specific set of parameters the resulting model of cantilever response was evaluated using the following cost function:

$$
J=\left\langle\left(S_{q q}-S_{y y}\right)^{2}\right\rangle,
$$

where $S_{q q}$ is the PSD of the modeled thermal response for a given set of parameters and $S_{y y}$ is the PSD of the measured thermal response of a microcantilever. The MATLAB function FMINSEARCH was used to minimize the cost function $J$ by adjusting the fitting parameters. The frequency range of the data selected for fitting captured enough of the peak so that the ratio of the maximum value in the selected data range to the minimum value in the selected data range was equal to 5 . While a value of 5 was selected arbitrarily, results calculated using data ranges with max-to-min ratios as small as 2 yielded similar results. An example curve fit is shown in Fig. 1 along with SHO and Sader model fits for comparison.

\section{Mapping the cost function}

The cost function $J$ presented in Eq. (12) serves as a means to compare the modeled cantilever response for a given set of parameters with measured data recorded for a specific cantilever. For a number of cantilevers, this cost function was evaluated at points over large ranges of $\omega_{n}$ and $\gamma$. For each pairing of $\omega_{n}$ and $\gamma$ values, the MATLAB function FMINSEARCH was used to scale the resulting model to 


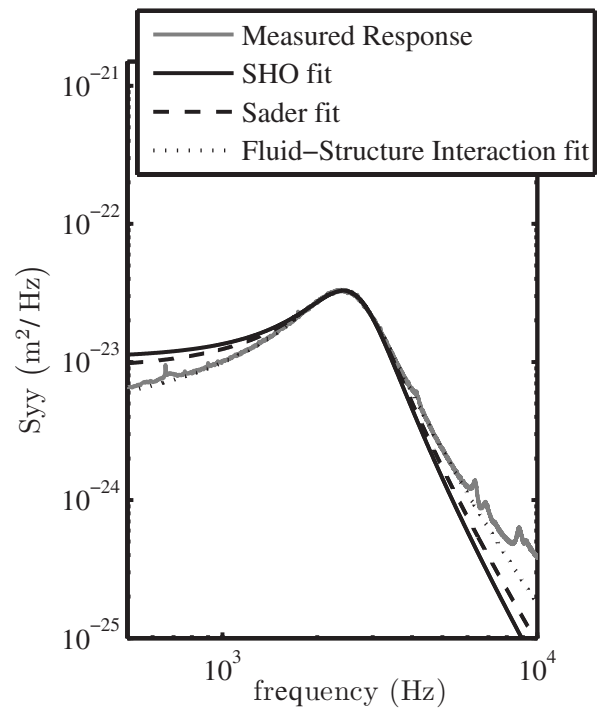

FIG. 1. The measured thermal vibration of MikroMasch cantilever model CSC38 B in water with SHO, Sader, and fluid-structure interaction model curve fits.

minimize the cost function. In this way, plots were created that mapped the cost as a function of $\omega_{n}$ and $\gamma$. An example plot is presented as Fig. 2.

\section{Heavy fluid loading calibration technique}

Based on the results of the initial data fitting effort and observations made by plotting the cost maps which will be discussed in Sec. V, a MATLAB program was written to automate the process of calibrating cantilevers by fitting the fluid-structure interaction model to a measured cantilever response in a way that restricted the fit to the heavy fluid loading region of the cost map. A flowchart of this method is presented in Fig. 3. The program first requires the user to provide initial parameter guesses to create a rough curve fit to the data. The program then identifies the frequency range of the data to be fit as was done in the unrestricted data fittings. The program then performs a series of curve fits to find points that lie on the low cost function curve. Points are identified along the curve starting from a low frequency and

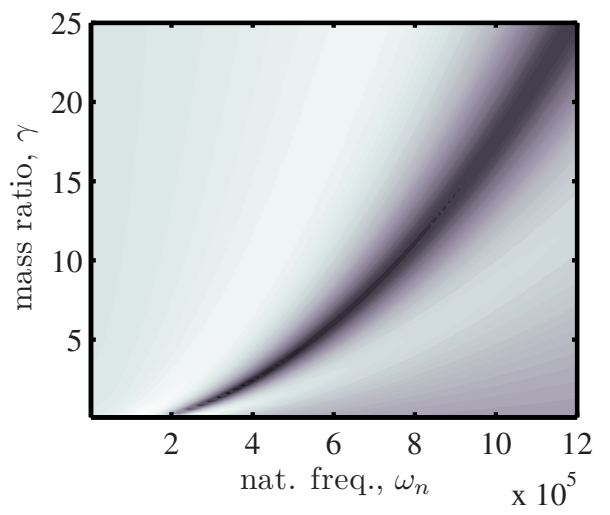

FIG. 2. (Color online) The cost function map for Veeco cantilever model ORC8 C in water identifying $\gamma$ and $\omega_{n}$ parameter values that result in fluidstructure interaction models that fit the measured data well. The shading scales with the cost function $J$, so that the darker regions of the map indicate a good fit.

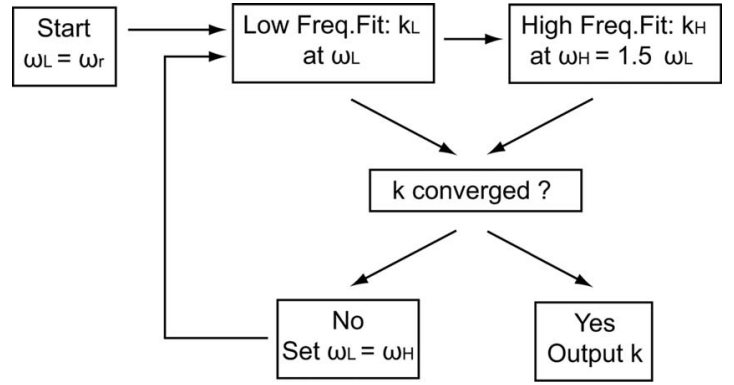

FIG. 3. A flowchart illustrating the calibration method. At each iteration, stiffness estimations are made at a low and a high frequency point along the low cost function curve. For each subsequent iteration, the previous high frequency point becomes the new low frequency point and a new high frequency point is selected. The resulting stiffness estimations at each iteration are compared to determine when the process has converged.

then at increasing frequencies until the resulting value for $k$ converges. To do this, the initial guess for $\omega_{n}$ is set to $\omega_{r}$. The program then uses the FMINSEARCH function to find the value of $\gamma$ and the scaling factor that best fits the model to the data for that specific value of $\omega_{n}$. This process is then repeated with a new $\omega_{n}$ guess that is $50 \%$ higher than the previous guess. A value for $k$ is found for each set of parameters using the following relation:

$$
k=\frac{3 m^{\prime} \omega_{n}^{2}}{\left(\alpha_{1} L\right)^{4} \gamma} .
$$

Each sequential set of parameters identifies a point along the low cost function curve with a higher mass ratio $\gamma$ than the previous point. In this way, the heavy fluid loading calibration program walks along the low cost function curve until the resulting value for $k$ converges, and is therefore not sensitive to the specific values of $\gamma$ and $\omega_{n}$ identified as will be discussed in Sec. V. A copy of the MATLAB program has been made available. ${ }^{32}$

\section{RESULTS AND DISCUSSION}

The calibration algorithm presented here was based on the results of the analysis of the cost function maps described in Sec. IV. For this reason, the findings from the cost function map investigation are presented here along with the results of the actual calibration technique.

\section{A. Cost function maps}

To better understand the stiffness estimates generated by an unrestricted fitting of the fluid-structure interaction model to measured data, MATLAB was used to map the cost function over a range of $\gamma$ and $\omega_{n}$ values as discussed previously (Fig. $2)$. Each point on the cost function map corresponds to a specific set of potential values of $\gamma$ and $\omega_{n}$. Dark regions of the plot identify a locus of parameters that yield fluidstructure interaction models that line up well with the measured cantilever frequency response. Each set of values for $\gamma$ and $\omega_{n}$ can be used to solve for a cantilever stiffness value. While accurate estimations of $\gamma$ and $\omega_{n}$ for a specific cantilever would yield accurate cantilever stiffness estimations, the imperfections of the model can cause the cost function to be minimized for incorrect values of $\gamma$ and $\omega_{n}$. 


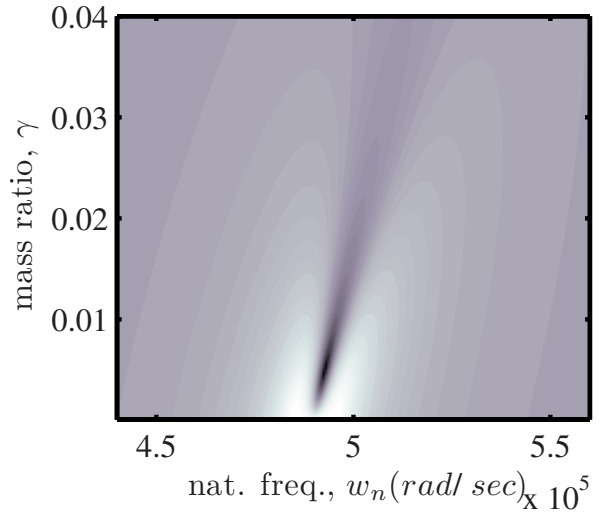

FIG. 4. (Color online) The cost function map for Veeco cantilever model ORC8 $\mathrm{C}$ in air rather than water.

The cost map looks different for cantilevers under light fluid loading. Figure 4 shows a cost function map for a cantilever in air. The low cost function locus is contained to a specific point. Under heavy fluid loading (see Fig. 2) the low cost function locus forms a curve establishing a relationship between $\gamma$ and $\omega_{n}$. Any set of $\gamma$ and $\omega_{n}$ values falling within this locus will yield a reasonable model of the cantilever dynamics. This reflects the findings that under light fluid loading, parameter identification is straightforward, while under heavy fluid loading, the fluid-structure interaction model is robust to parameter uncertainty, which makes curve fitting based parameter identification difficult. ${ }^{33}$

Overlaying curves of the $\gamma$ and $\omega_{n}$ points that yield constant cantilever stiffness values onto a cost function map can illustrate the sensitivity of the resulting stiffnesses to changes in $\gamma$ and $\omega_{n}$ values. It is also illustrative to scale the abscissa against $w_{n}^{2}$ to linearize the curves of constant stiffness, as is shown in Fig. 5. The low cost function locus aligns with the lines of constant stiffness for high values of $\gamma$ and $\omega_{n}$. This indicates that for higher values of $\gamma$ and $\omega_{n}$ within the locus, the resulting stiffness is not sensitive to perturbations. If the mass loading describing the cantilever behavior is sufficiently high, the cantilever stiffness estimation is not sensitive to changes in the $\omega_{n}$ and $\gamma$ estimations as long as the fluid-structure interaction model provides a good fit to the

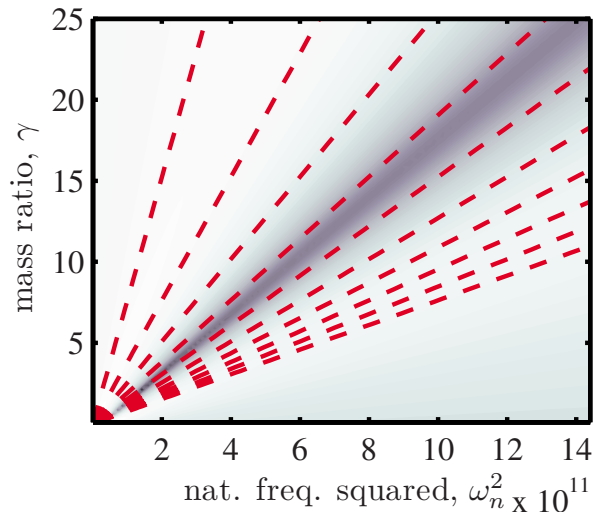

FIG. 5. (Color online) The cost function map for Veeco cantilever model ORC8 C in water plotted against $\omega_{n}^{2}$ rather than $\omega_{n}$. Red dashed lines indicate $\gamma$ and $\omega_{n}$ values that yield constant stiffness estimates. The lines were generated for stiffnesses ranging from $k=0.1 \mathrm{~N} / \mathrm{m}$ to $k=1.0 \mathrm{~N} / \mathrm{m}$ stepping in $0.1 \mathrm{~N} / \mathrm{m}$ increments.

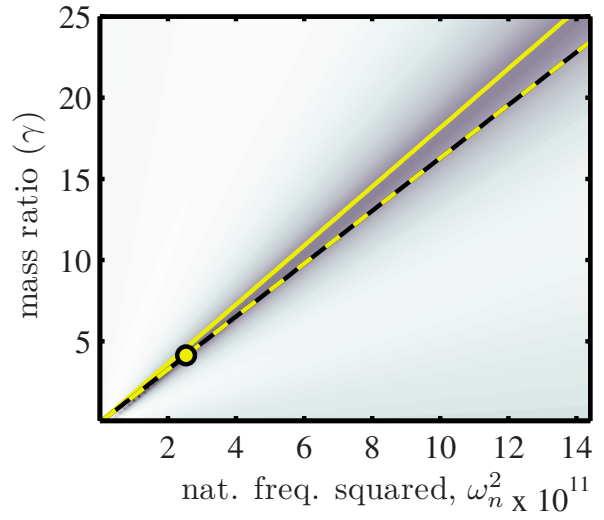

FIG. 6. (Color online) The cost function map for Veeco cantilever model ORC8 $\mathrm{C}$ in water. The circle indicates the unrestricted minimum point of the cost function. The dashed line corresponds to the stiffness estimated by the unrestricted data fitting while the solid line indicates the points that correspond to the stiffness estimated using the new heavy fluid loading algorithm. This line remains within the low cost locus for higher values of $\gamma$.

data using those parameters. This implies that in these cases, cantilevers can be calibrated under heavy fluid loading by fitting the fluid-structure interaction model to data in a way that restricts the search to the high $\gamma$ region of the locus. While it is difficult to identify the individual $\omega_{n}$ and $\gamma$ parameters from the thermal response of a heavily loaded cantilever, ${ }^{33}$ this technique only requires the identification of the ratio of $\omega_{n}^{2}$ to $\gamma$. This can be seen in Eq. (13), where identifying parameters $m^{\prime}, \alpha_{1}$, and $L$ only requires knowledge of the fluid density and the plan-view dimensions of the cantilever beam. The effect of searching for the appropriate ratio of $\omega_{n}^{2}$ to $\gamma$ is to reduce two degrees of freedom in the fitting process to a single degree of freedom.

To illustrate the difference between the unrestricted curve fitting and the new technique presented here, the $\omega_{n}$ and $\gamma$ values corresponding to an unrestricted curve fitting have been identified on a cost map in Fig. 6. The line running through this point indicating the values of $\omega_{n}$ and $\gamma$ that would yield the same stiffness can be seen to diverge from the low cost function locus. That indicates that the resulting stiffness estimate from the unrestricted curve fitting is sensitive to perturbations to the fit parameters. This is undesirable since there is significant uncertainty involved with the curve fitting of cantilevers under heavy fluid loading. ${ }^{33}$ The second line identifies parameter combinations that result in the stiffness estimate generated by the algorithm presented here. It runs along the low cost function locus for high fluid loading values identifying a range of parameters that yield good fits between the fluid-structure interaction model and the measured data.

\section{B. Heavy fluid loading curve fit results}

Calibrations were performed in air using Sader's method and the thermal noise method and in de-ionized water using the thermal noise method, the unrestricted fitting of the FSI model, and using the new restricted FSI model fitting algorithm presented here. The results are presented in Table I. To illustrate the difference between each calibration method and 
TABLE I. Comparison of stiffness estimations for 11 different cantilever models. The ORC 8 and RFESP cantilevers were manufactured by Veeco, while the CSC38 and NSC36 cantilevers were manufactured by MikroMasch. The methods used include Sader's method, an unrestricted fitting of the fluid-structure interaction model to data, the new method presented in this document, and the thermal noise method. The values for stiffness $k$ are in $\mathrm{N} / \mathrm{m}$. The length-to-width ratio $L / b$ is assumed to be much greater than 1 in the fluid loading model, which impacts all methods here except for the thermal noise method.

\begin{tabular}{cclllllr}
\hline \hline No. & Model & $k_{\text {Sader,air }}$ & $k_{\text {FSI fit,water }}$ & $k_{\text {new,water }}$ & $k_{\text {therm,air }}$ & $k_{\text {therm,water }}$ & $L / b$ \\
\hline 1 & ORC8 A & 0.848 & 0.970 & 0.961 & 0.885 & 0.827 & 2.6 \\
2 & ORC8 B & 0.127 & 0.137 & 0.136 & 0.125 & 0.131 & 5.1 \\
3 & ORC8 C & 0.467 & 0.486 & 0.455 & 0.564 & 0.544 & 5.2 \\
4 & ORC8 D & 0.0674 & 0.0708 & 0.0682 & 0.0709 & 0.0752 & 10.2 \\
5 & CSC38 A & 0.158 & 0.175 & 0.172 & 0.128 & 0.145 & 6.9 \\
6 & CSC38 B & 0.0494 & 0.0514 & 0.0491 & 0.0429 & 0.0467 & 10.6 \\
7 & CSC38 C & 0.0802 & 0.0883 & 0.0868 & 0.0713 & 0.0808 & 8.4 \\
8 & NSC36 A & 2.23 & 2.69 & 2.52 & 2.11 & 2.01 & 3.0 \\
9 & NSC36 B & 4.69 & 5.68 & 5.53 & 4.85 & 4.26 & 2.4 \\
10 & NSC36 C & 1.48 & 1.71 & 1.62 & 1.38 & 1.30 & 3.6 \\
11 & RFESP & 5.76 & 6.29 & 5.94 & 4.35 & 4.03 & 5.0 \\
\hline \hline
\end{tabular}

Sader's method, the percent difference was plotted for each cantilever in Fig. 7.

Since the fluid loading model assumes that the cantilever length greatly exceeds the cantilever width, the results in Fig. 7 are ordered according to the $L / b$ ratios such that from left to right, $L / b$ values decrease, and therefore this assumption becomes less valid. In the initial presentation of Sader's method, ${ }^{14}$ accuracy is claimed for cantilevers with $L / b$ ratios that exceed 3. These results find this new method performed in water to be within $10 \%$ of Sader's method performed in air for $L / b$ values that exceed 3.5 (which is indicated by the vertical gray line in Fig. 7). This may be an indication that the $L / b$ requirement plays a larger role under heavy fluid loading (which is an intuitive result).

The restricted FSI fitting calibration technique can be seen to provide a closer stiffness value to Sader's method than the unrestricted FSI fitting for all cases tested. For the eight cantilevers with $L / b>3.5$, the absolute improvement

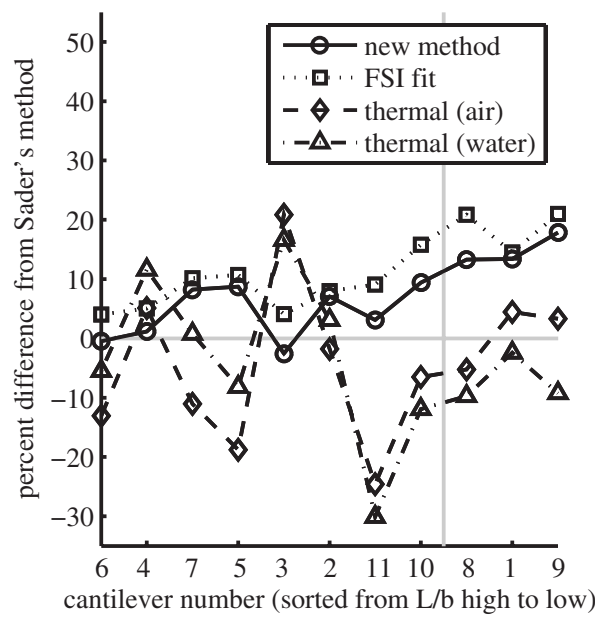

FIG. 7. The percentage difference between each calibration value and the calibration determined via Sader's method for each cantilever. The cantilevers are ordered according to their $L / b$ ratio and are numbered to match the labels in Table I. Cantilevers to the right of the vertical gray line have $L / b$ ratios less than 3.5 . ranged from $0.9 \%$ for cantilever 2 to $6.4 \%$ for cantilever 10 . The average improvement was $3.4 \%$. In three cases (cantilevers 5,7 , and 10), the new method improved the calibration from a difference larger than $10 \%$ to a difference smaller than $10 \%$.

The calibrations produced using the thermal noise method are included in Table I and Fig. 7 for comparison. The thermal noise calibrations performed in air and in water varied from the Sader's method calibrations and from each other over a range up to the typical $20 \%$ (with the exception of cantilever 11, which exhibited a poor signal-to-noise ratio during testing). The selection of Sader's method to serve as the standard to plot against should not be interpreted as an indication of the superior accuracy of one method over another. The cantilevers used in this study were pointed or "dog eared" at the free end rather than rectangular. This is a common feature of commercially available cantilevers that are not accounted for in the fluid loading model and could contribute to inaccuracy. Additionally, the shape of the free end of the cantilever could impact the $\chi$ value, which would affect the comparison of intrinsic stiffnesses to effective stiffnesses. The thermal noise data collected for the higher stiffness cantilevers (cantilevers 9 and 11) exhibited weaker signal-to-noise ratios which may impact the thermal noise calibration results, as has been noted elsewhere. ${ }^{7}$ For these reasons, further study is warranted into the accuracy of various calibration techniques under various conditions. The results presented here suggest that the restricted FSI fitting technique can serve as a tool to pursue this line of study.

\section{SUMMARY AND CONCLUSIONS}

A new cantilever calibration technique has been presented that is specifically designed for calibrating rectangular cantilevers by analyzing the thermal response under heavy fluid loading. This technique is similar to Sader's calibration method in two ways: it does not require absolute magnitude scaling of the thermal vibration measurement (i.e., it does not require photodiode calibration for optical lever-type 
AFM measurements) and it requires only knowledge of the length and width of a cantilever along with the density and viscosity of the surrounding fluid. This technique was used to calibrate 11 cantilevers (six MikroMasch and five Veeco cantilevers) that covered a stiffness range from 0.05 to $5.8 \mathrm{~N} / \mathrm{m}$. For comparison, the cantilevers were also calibrated in air using Sader's method. For cantilevers with $L / b$ ratios greater than 3.5, then the maximum difference between Sader's method performed in air and this new method performed in water was $9.4 \%$. A MATLAB program that performs this calibration has been made available.

\section{ACKNOWLEDGMENTS}

The authors would like to thank Dr. Monica Rivera for designing and building the AFM used in some of the preliminary work in this effort and the National Science Foundation, which funded this research through the Integrative Graduate Education and Research Traineeship program (Grant No. DGE-0221632).

${ }^{1}$ J. D. Holbery, Rev. Sci. Instrum. 71, 3769 (2000).

${ }^{2}$ N. A. Burnham, Nanotechnology 14, 1 (2003).

${ }^{3}$ C. A. Clifford, Nanotechnology 16, 1666 (2005).

${ }^{4}$ S. B. Aksu, B. Saltuk, J. A. Turner, and A. Joseph, Rev. Sci. Instrum. 78, 043704 (2007).

${ }^{5}$ R. S. Gates and M. G. Reitsma, Rev. Sci. Instrum. 78, 086101 (2007).

${ }^{6}$ G. Y. Jing, J. Ma, and D. P. Yu, J. Electron Microsc. 56, 21 (2007).

${ }^{7}$ E. D. Langlois, G. A. Shaw, J. A. Kramar, J. R. Pratt, and D. C. Hurley, Rev. Sci. Instrum. 78, 093705 (2007).
${ }^{8}$ G. V. Lubarsky and G. Hhner, Rev. Sci. Instrum. 78, 095102 (2007).

${ }^{9}$ B. Ohler, Rev. Sci. Instrum. 78, 063701 (2007).

${ }^{10}$ Z. C. Ying, M. G. Reitsma, and R. S. Gates, Rev. Sci. Instrum. 78, 063708 (2007).

${ }^{11}$ J. P. Cleveland, Rev. Sci. Instrum. 64, 403 (1993).

${ }^{12}$ P. J. Cumpson, Nanotechnology 14, 918 (2003).

${ }^{13}$ L. J. Hutter and J. Bechhoefer, Rev. Sci. Instrum. 64, 1868 (1993).

${ }^{14}$ J. E. Sader, J. W. M. Chon, and P. Mulvaney, Rev. Sci. Instrum. 70, 3967 (1999).

${ }^{15}$ S. M. Cook, T. E. Schaffer, K. M. Chynoweth, M. Wigton, R. W. Simmonds, and K. M. Lang, Nanotechnology 17, 2135 (2006).

${ }^{16}$ R. Proksch, J. P. Cleveland, M. B. Viani, and R. C. Callahan, "Look ma, no hands: Calibrating spring constants without touching a surface" (http:// www.asylumresearch.com/applications/cantilevercal/stmpresentation.pdf).

${ }^{17}$ A. W. McFarland, Appl. Phys. Lett. 87, 053505 (2005).

${ }^{18}$ M. J. Lachut and J. E. Sader, Phys. Rev. Lett. 99, 206102 (2007).

${ }^{19}$ G. U. Lee, L. A. Chrisey, and R. J. Colton, Science 266, 771 (1994).

${ }^{20}$ R. Proksch, T. E. Schaffer, J. P. Cleveland, R. C. Callahan, and M. B. Viani, Nanotechnology 15, 1344 (2004).

${ }^{21}$ M. J. Higgins, Rev. Sci. Instrum. 77, 013701 (2006).

${ }^{22}$ H. J. Butt, Nanotechnology 6, 1 (1995).

${ }^{23}$ J. E. Sader, J. Appl. Phys. 84, 64 (1998).

${ }^{24}$ M. R. Paul, Phys. Rev. Lett. 92, 235501 (2004).

${ }^{25}$ D. G. Cole and R. L. Clark, J. Appl. Phys. 101, 034303 (2007).

${ }^{26}$ W. T. Thomson, Theory of Vibration with Applications (Prentice-Hall, Englewood Cliffs, NJ, 1972).

${ }^{27}$ A. Einstein, Ann. Phys. 322, 549 (1905).

${ }^{28}$ F. E. Gittes, Eur. Biophys. J. 27, 75 (1998)

${ }^{29}$ P. Attard, Rev. Sci. Instrum. 77, 116110 (2006).

${ }^{30}$ D. A. Walters, J. P. Cleveland, N. H. Thomson, P. K. Hansma, M. A. Wendman, G. Gurley, and V. Elings, Rev. Sci. Instrum. 67, 3583 (1996).

${ }^{31}$ J. L. Hutter, Langmuir 21, 2630 (2005).

${ }^{32}$ The calibration program is available for download at code.google.com $/ \mathrm{p} /$ afmcalibration.

${ }^{33}$ D. G. Cole, Meas. Sci. Technol. 19, 125101 (2008). 